\title{
Analysis of Writing Strategies on English Abstract of Medical Academic Paper
}

\author{
Fei Chen, Cheng Chen* \\ School of Humanities and Information Management, \\ Chengdu Medical College, \\ Chengdu, China \\ *Correspondence: 183256422@qq.com
}

\begin{abstract}
English abstracts of medical literature influence international communication of medical information. Problems in English abstracts of domestic papers have seriously prevented domestic medical achievements from being recognized and identified internationally. In order to figure out features of sentence structure and textual organization of medical English abstract, the paper firstly analyzed, in term of textual function, some sample abstracts-English abstracts of international distinguished medical academic papers. The textual analysis conducted quantitatively and qualitatively from the following aspects: thematic structure and thematic progression pattern. And then, based on the analytic results, writing strategies and guidelines were proposed in detail in order to help medical college students write high-quality English abstracts which can meet the international standard. The study suggests a simple theme, simple structure and constant theme progression pattern are dominantly employed in medical academic writing.
\end{abstract}

Keywords-writing strategies; English abstract writing; medical academic paper; textual function

\section{INTRODUCTION}

In the age of globalization, information exchange in the field of medicine goes beyond country boundaries. In that process, English abstracts play a vital role. The abstract, a condensed representation of the contents of an article, provides readers with a concise knowledge of the full article and even decides whether the paper can be accepted and published by periodicals. However, the quality of English abstracts in Chinese medical academic papers is not satisfactory, let alone exerts influences internationally. Therefore, it is essential to put forward corresponding solutions for high-quality English abstracts. With the increasing importance of English abstracts, studies on them are flourishing. Researchers have proposed writing techniques on not only the micro-features of abstracts, but also the macro-genre structure. Swales and Feak illustrated the two general ways to write a well-organized research paper abstract in 1994 [1]. Slade, in 2000, provided the essential contents that should be included in an abstract [2]. Slalager-Meger in 1990 focused the study on the rhetorical structure of medical English abstract [3]. However, it has been noticed that studies on the language of abstracts are rare. Language and text organization are the two keys to a successful abstract. Textual

Sponsored by Sichuan Foreign Language and Literature Research Center: A Corpus-based Study of English Abstracts of International Medical Academic Papers from Perspective of Functional Linguistics (No. SCWYH14-05) function of Systemic Functional Linguistics (SFL), which analyzes not only the language form but also the text organization, is taken as the theoretical guide of the study. The framework of thematization can facilitate us to get a better understanding of the organization mode of a text and how information is transmitted and thus can help organize English abstract in a more native and appropriate way. In this study, 50 English abstracts of international distinguished medical academic papers were chosen as the research samples from periodicals with an influential index higher than 10 from PubMed published from 2014 to 2018, with 10 abstracts from each year. After then the author made a qualitative and quantitative analysis of these abstracts in term of textual function from two aspects: thematic structure and thematic progression pattern. For thematic structure, the analysis was conducted from the perspectives of theme types, and markedness of theme. Finally, abstract writing features and strategies were summarized and proposed on the basis of the research results.

\section{THEORETICAL BACKGROUND}

\section{A. Abstract Types}

The abstract is a discourse, independent of the article in form but closely related to it in content. It offers concise knowledge of the paper. According to the content, abstracts can be divided into three types: informative abstract, indicative abstract and informative-indicative abstract. Informative abstracts inform readers of such comprehensive information of the paper briefly as research purpose, method, data and conclusion. Such abstracts are usually framed in a fixed structure made up of the previous parts. Indicative abstracts simply tell readers the main topic discussed in the paper without any mention of research process, and evidence. Such abstract can help readers to decide whether it is worthy of further reading of the full article. The last type is the combination of the two. For the most important part, it is informative, while the other parts are indicative [4].

\section{B. Textual Function of SFL}

Halliday (2000) points out all English clauses include two parts-Theme and Rheme [5]. Theme is the element which serves as the point of departure of the message. He further puts Theme into four different categories-Simple Theme, Multiple 
Theme, Clausal Theme and Predicated Theme. Simple Theme refers to the theme that consists of one or more groups or phrases, forming just one structural element. It always represents an experiential element of ideational function (such as participant, process and circumstantial elements), and consequently, it is also called an experimental theme or topical theme. Experimental theme can usually be realized by nominal groups, adverbial groups, and prepositional groups. Multiple Theme includes more than one theme, with one representing experimental function and the other interpersonal or textual function or both. Experimental theme follows textual and/or interpersonal theme. The predicated theme is known as "cleft sentence" in formal Grammar-it is...that... When a clause serves as the theme of a clause complex, this clause is regarded as clausal theme. A maximally extended example of Multiple Theme is shown below to demonstrate the sub-categories [5].

Well, but then, surely wouldn't the best idea be to join the group.

The two words: but and then, belong to textual theme, showing textual relationship of transition. Well, surely and wouldn't belong to interpersonal theme, indicating the speaker's personal attitude and the best idea is the experimental theme, showing the departure point of the information and the topic under discussion.

Each sentence has its own thematic structure. A text includes more than one sentence. The relationship between successive themes is called as the progression pattern. Danes (1984) defines it as the choice and ordering of utterance theme and their relationship to the whole text [6]. It organizes the development of the text from semantic coherence. Many scholars such as Guowen Huang (1988) [7], and Yongsheng Zhu (1995) have all made great contributions to the discussion of thematic progression (TP) patterns [8]. In this study, the progression patterns proposed by Yongsheng Zhu are adopted, which consist of four types: simple linear (SL), constant theme (CT), constant rheme (CR) and cross-progression (CP). CT shows the same theme occurs but rhemes are different in a series of sentences. CR is a kind of pattern in which a series of sentences use the same theme but different themes. SL means the rheme or one part of the rheme of one sentence becomes the theme of the following sentence. CP, contrary to $\mathrm{SL}$, means the theme of the previous sentence turns into the rheme or one part of the rheme of the following sentence.

\section{ANALYSiS OF WRITING FEATURES OF SAMPLE ABSTRACTS}

The author of this paper interpreted and analyzed quantitatively the corresponding number of sentences and their corresponding percentages in each theoretic field. The results were further explained qualitatively. In total, there are 450 sentences in the 50 English sample abstracts. Analysis on the theme types of thematic structure is initially conducted. Then, the markedness of theme is analyzed. Progression pattern is the last subject under discussion.

\section{A. Analysis of Theme Types}

The author firstly decided the theme of each sentence, and then analyzed which type each theme belongs to. The number of the same type is caculated and so is the proportion. The results are shown below.

TABLE I. ANALYSIS OF THEME TYPES

\begin{tabular}{|c|c|c|}
\hline Types & Number & Percentage \\
\hline Simple Theme & 295 & $65.56 \%$ \\
\hline Multiple Theme & 140 & $31.11 \%$ \\
\hline Clausal Theme & 15 & $3.33 \%$ \\
\hline
\end{tabular}

From the data, we can know Simple Theme takes up a dominant proportion in abstracts, and Multiple Theme follows next. Clausal Theme is seldom used, and no Predicated Theme is noticed. Among the three types of realization of experimental theme: nominal group, adverbial group, and prepositional group, in these abstracts, themes are mostly realized by nominal groups, the words indicating the topic of the abstract.

As the second widely used theme, Multiple Theme is worthy of our further analysis to gain the distributions of the textual and interpersonal themes. Halliday points out Multiple Theme can be expressed in the following formulas: textual theme + experimental theme; interpersonal theme+ experimental theme; textual theme + interpersonal theme+ experimental theme, so next the study figures out the number of each formula and its proportion.

\section{B. Analysis of Semantic Coherence}

TABLE II. ANALYSIS OF MULTIPLE THEME FORMULAS

\begin{tabular}{|c|c|c|}
\hline Types & Number & Percentage \\
\hline Textual theme + experimental theme & 123 & $87.86 \%$ \\
\hline Interpersonal theme+ experimental theme & 17 & $12.14 \%$ \\
\hline $\begin{array}{c}\text { Textual theme + interpersonal theme + } \\
\text { experimental theme }\end{array}$ & 0 & $0 \%$ \\
\hline
\end{tabular}

The results indicate "textual theme + experimental theme" occupies the largest proportion, which greatly outnumbers the other two types. Textual theme serves for the coherence of the text. It functions to help readers follow the organization of the text. Interpersonal theme expresses the writer's comments or judgments. An academic writing, naturally aiming at stating an idea, is impersonal. Consequently, it is natural to get the result. Halliday expresses that textual theme is mainly realized by any combination of continuative themes, conjunctions and conjunctive adjuncts, and it shows how the clause is related to the preceding one, and sets up not only grammatical relationship but also semantic relationship between clauses [5]. Conjunctions are items which relate the clause to the preceding clause in the same sentence while conjunctive adjuncts are those which relate the sentence to the preceding text, such as "therefore", "in other words", "so far", etc. They both are used to show different semantic concepts such as time, concession, cause and so on. To make clear how sentences are related to each other in medical English abstracts, the full details of the 
components of the textual theme would be specified next. Because continuative themes are mainly used in oral English, the specification is only confined to the other two components. The following table shows the number and proportion of the two components.

TABLE III. SPECIFICATION OF TEXTUAL THEME

\begin{tabular}{|c|c|c|}
\hline Types & Number & Percentage \\
\hline Conjunctions & 20 & $16.26 \%$ \\
\hline Conjunctive adjuncts & 103 & $83.74 \%$ \\
\hline
\end{tabular}

Table III shows textual theme is realized more by conjunctive adjuncts, especially ones showing concept of addition, such as "in addition", "furthermore" and etc. Conjunctions show relationship within one sentence, while conjunctive adjuncts show relationship between sentences. In other words, conjunctions are usually used to form complex sentences to show the semantic relationship between clauses within a sentence and conjunctive adjuncts show the semantic relationship between sentences.

The result that conjunctive adjuncts outnumber conjunctions implies that simple sentences are more preferable than complex sentences in such abstracts. Actually, this conforms to the fact that medical English abstracts of academic papers are informative, intending to inform readers of significant information such as an advance or progress in medical field. Brief and concise sentences can help readers understand and appreciate the paper better.

\section{Analysis of Subject Selection}

Both in a Simple Theme and Multiple Theme, experimental element is indispensable. Experimental theme can be further divided into marked and unmarked themes. Each component of a clause has its own fixed position. For example, in a declarative clause, the subject is grammatically put at the beginning of the clause, and thus the subject is the experimental theme. Such theme is unmarked theme. However, sometimes, in order to emphasize other components instead of subject, the writer purposely puts the components at the beginning of the clause and thus makes it the experimental theme, and such theme is marked. In other words, when the theme mapped onto the subject, the theme is unmarked. Otherwise, it is marked. The following are examples of marked and unmarked themes.

E.g. 1. Drugs (theme) are tested in clinical trials on genetically diverse patient populations.

E.g. 2. In zebrafish (theme), DNA methylation patterns are programmed in transcriptionally quiescent cleavage embryos.

The above examples show that the theme of the first sentence-drugs are an unmarked theme because it also serves as the subject. The theme of the second sentence is marked because it grammatically serves as the adverbial adjunct of the sentence, not the subject. The following discusses the makedness of theme to see how the information is organized and emphasized.
TABLE IV. FREQUENCY OF MARKED AND UNMARKED THEMES

\begin{tabular}{|c|c|c|}
\hline Types & Number & Percentage \\
\hline Marked theme & 135 & $30 \%$ \\
\hline Unmarked theme & 315 & $70 \%$ \\
\hline
\end{tabular}

Halliday (2000) has pointed out that adjuncts and complements are the main tendencies for the selection of marked theme[5]. From the above example and data, we can see that among the marked theme, most of them are practically adjuncts. Marked theme is used for emphasis. When the information is needed to be emphasized, it can be put at the beginning of a sentence to be a marked theme. It is not used at random as we please.

\section{Analysis of Textual Organization}

TP patterns do play an important role in the textual organization so it is quite essential to conduct a study on it. In TP patterns, theme is limited to experimental theme which actually carries essential textual information. In the process of analysis, two principles are complied with. The analysis is carried out only between two adjacent sentences. Additionally, TP patterns beyond the discussed types are out of our consideration. In SL and CP patterns, the exchange between theme and rheme does not mean they must be exactly the same words. Instead, it can also be achieved by synonymous expressions. In a word, they are identical in sense but not in form. The finding is shown in the following table.

TABLE V. DISTRIBUTION OF TP PATTERNS

\begin{tabular}{|c|c|c|}
\hline Types & Number & Percentage \\
\hline SL & 10 & $2.23 \%$ \\
\hline CT & 334 & $74.39 \%$ \\
\hline CR & 101 & $22.49 \%$ \\
\hline CP & 4 & $0.89 \%$ \\
\hline
\end{tabular}

The table suggests that SL and CP take up such a low proportion that they can even be ignored. CT pattern is the most frequently used as much as $74.39 \%$ and CP follows next. The result is attributed to the nature of medical academic paper. Such abstracts mainly tell the readers what topic the paper is concerned about. All the other sentences are constructed to elaborate the topic clearly and thoroughly with emphasis put on the topic being talked about. Consequently, the same theme is adopted in sentences.

\section{STRATEGIES ON ENGLISH ABSTRACT WRITING}

A study of textual function of sample abstracts of outstanding international medical research papers demonstrates international standards of high-quality English abstract. To keep in line with international standards, efforts should be made in the following areas.

To begin with, abstract of medical paper belongs to indicative type, which aims at introducing to readers such topics as medical achievements, progress or a new cure approach. What matters is not how such achievements are accomplished and processed, but the topic information, so other factors like method and process are not indispensable. In 
other words, such abstracts should be kept short, concise and clear. That can help readers have a quick and better appreciation of the information with ease. Moreover, Simple Theme is the most commonly used theme type, followed by Multiple Theme. That means simple sentence structure should be the first choice in composing a sentence.

In addition, most themes should be unmarked, which means the subject is usually put in the beginning and thus maps onto the experimental theme. Only when you want other components to be stressed, can they be put at the beginning of the sentence and make it marked. In other words, marked theme is employed only to show the author's desire to highlight some information or to expresses the author's construction of the idea and his certain particular intention. For example, when the temporal clause serves as the marked theme, it reveals that the author wants to attract readers' attention to the time instead of to what. In a word, marked theme is not used at random but instead, it serves for the information and the author's intention, and thereby the information and the author's intention can be conveyed accurately and clearly. However, some Chinese authors tend to follow our traditional spiral ways of thinking and put the adverbial clauses at the beginning of the sentence, and thus make it marked theme. That should be paid special attention to and avoided in writing. If the marked theme is over-used, it may cause confusion and readers cannot know what the key point is.

Thirdly, interpersonal theme, which indicates the author's attitude or opinion, is rarely used. It means words that show modality and mood should be avoid being used, such as "can", "must", "absolutely" and "definitely" and so on. Conjunctive adjuncts instead of conjunctions are more employed to show semantic relationship between sentences. Among the adjuncts, the adjuncts showing concept of addition, such as "furthermore", "in addition", and so on are frequently used to display the core information. The fact that conjunctions are less used also indicates that complex structures are not quite welcomed in such abstracts.

Last, CT progression pattern is the most widely used pattern for the information organization. Such abstracts develop and center on one topic and the words of the topic are constantly used as the theme. In other words, in writing, the words of the topic should be initially chosen as the subject and made as the theme.

\section{CONCLUSION}

The study explores the organizational ways of English abstracts of medical academic papers, summarizes the main features of such abstracts and provides some writing references for medical college students to help them to compile English abstract in a more accurate and acceptable way by the international community.

Abstract of medical academic paper belongs to indicative type whose ultimate purpose is to convey topic information to readers. Therefore, it is unnecessary to do the writing in a long and detailed discussion. Keeping it short and clear is the key.

Additionally, when it comes to sentence structure, principle of simplicity is what students should stick to. Simple sentence structure is the first choice. And that's why semantic relationship is realized more by conjunctive adjuncts than conjunctions.

Finally, in such abstracts, the topic of the paper can be chosen as the subject, and in most cases, it maps onto the theme. In other words, the subject is usually placed at the beginning of sentences. Chinese students should especially resist against the traditional spiral ways of thinking: adverbial clauses comes first. Do not place other components at the beginning of a sentence casually unless other components are to be emphasized. Which component is chosen as the theme depends on the author's construction of the idea and his certain particular intention.

In conclusion, all these strategies can help students to write an excellent English abstract. A good English abstract can open the door to international medical circle for the medical college students, make the achievements known by the world and promote medical exchange on a global level.

\section{ACKNOWLEDGMENT}

Firstly, I would like to give my deepest and sincerest appreciation to my superior Xuecheng Wang. Thank him for his encouragement, support and help. His help and encouragement assist me to overcome the difficulties in my writing and finally to accomplish my paper.

Secondly, I want to show my gratitude to my colleges for their valuable suggestions, especially Cheng Chen. I was inspired and provided with rich and useful materials which help me conduct my study. Thank them all again!

\section{REFERENCES}

[1] J. M. Swales and C.B Feak, Academic Writing for Graduate Students: Essential Tasks and Skills. Ann Arbor: The University of Michigan Press, 1994. (references)

[2] C. Slade, Form and Style: Research Papers, Reports and Thesis. Beijing: Foreign Language Teaching and Research Press, 2000. (references)

[3] F. Slalager-Meger, "Discoursal flaws in medical English abstracts," a genre analysis per research -and-text- type, No.4, pp.365-384, 1990.

[4] D. Sijia and H. Jie, "Analysis on problems in English abstract writing of academic paper-college graduates’ papers in Nei Menggu Normal University as examples,” Chinese Journal (foreign language education and teaching), No.5, pp.146-147, 2015. (In Chinese)

[5] M.A.K. Halliday, An Introduction to Functional Grammar, 2nd ed., Bei Jing: Foreign Language Teaching and Research Press, 2000. (references)

[6] F. Dane, Functional Sentence Perspective and the Organization of Text. The Hague: Mouton, 1974. (references)

[7] H. Guowen, Essentials of Discourse Analysis. Chang Sha: $\mathrm{Hu} \mathrm{Nan}$ education press, 1988. (In Chinese)

[8] Z. Yongsheng, "Theme progression pattern and discourse analysis," Foreign Language Teaching and Research, No.3, pp.7. 1995. (In Chinese). 